\title{
Cardiovascular safety of dipeptidyl peptidase-4 (DPP-4) inhibitors
}

\section{ABSTRACT}

Incretin-based agents, called gliptins or dipeptidyl peptidase-4 inhibitors (DPP-4), are currently considered a promising therapeutic option. It has been known for a long time that these drugs effectively improve glycaemic control, which is reflected by significant $\mathrm{HbA}_{1 \mathrm{c}}$ reduction, without increasing the rate of hypoglycaemia or body weight gain, and that they do not have significant adverse effects. Is it, however, enough to consider them as 'safe'? No, certainly not. Currently, when assessing the safety of glucose-lowering drugs, the effect on cardiovascular system must be also (and first of all) taking into account. Except for glucose-lowering activity, gliptins have multiple pleiotropic effects and, additionally, are safe in terms of cardiovascular risk, which was proved based on the results from large clinical trials such as SAVOR-TIMI, EXAMINE and, the most recent, TECOS. The future will show whether long-term follow-up of patients treated with gliptins and the results of further trials will confirm current knowledge about DPP-4 inhibitors and expectations associated with this class of drugs. (Clin Diabet 2015; 4, 6: 238-242)

Key words: DPP-4, cardiovascular safety, type 2 diabetes

\section{Introduction}

Despite progress in diagnostics and treatment, vascular complications of diabetes remain the most important problem of contemporary diabetology and significantly deteriorate the patients' quality of life. They contribute to increased diabetes-related morbidity and mortality, and, as a consequence, to reduced life expectancy compared with general population. It seems that chronic hyperglycaemia remains a key factor leading to development and subsequent progression of vascular complications, besides broad-sense environmental factors and a genetic predisposition. Despite treatment intensification, most patients with diabetes have poor metabolic control. Therefore, although there are many oral antidiabetic drugs and insulin preparations available nowadays, new therapies mimicking physiological mechanism are still searched for [1-4].

The observation that food ingestion or enteral glucose administration leads to significantly stronger stimulation of insulin secretion by pancreatic $\beta$-cells compared with intravenous glucose administration raised an interest in glucagon-like peptide-1 (GLP-1). This phenomenon is called incretin effect.

The incretin effect plays a key role in the regulation of glucose metabolism in healthy subjects and is responsible for about $50-70 \%$ of postprandial insulin response. This effect is mediated by several active peptides released by intestinal cells. Among these peptides, glucagon-like peptide-1 (GLP-1) and glucose-dependent insulinotropic peptide (GIP) play the most important role in the meal-induced insulin secretion. In healthy subjects, the release of GLP-1 in response to food ingestion occurs very quickly (within less than 10 minutes) and is correlated with insulin secretion into the portal circulation. In patients with type 2 diabetes or impaired glucose tolerance (IGT), this response is abnormal, which leads to reduced postprandial insulin secretion [5-8].

The treatment of diabetes must be individually tailored for each and every patient, both in terms of 
therapeutic target and its achievability. Various mechanisms leading to disease development should be taken into account. The treatment demands continuous and close cooperation with the patient and has to be multidirectional and consistent with current therapeutic guidelines. When adjusting individual treatment regimen, patient's quality of life should also be considered. Efforts to achieve blood glucose normalization as safely as possible is of key impotance in prevention and slowing down the progression of chronic complications of diabetes. On the other hand, the impact of intensive glicaemic control on macrovascular complication is disputable. Large clinical trials, ACCORD (Action to Control Cardiovascular Risk in Diabetes), ADVANCE (Action in Diabetes and Vascular Disease-Preterax and Diamicron Modified Release Controlled Evaluation) and VADT (Veterans Affairs Diabetes Trial), that were designed and performed to prove beneficial effect of intensive treatment on reduction of cardiovascular events in patients with type 2 diabetes, failed to show any reduction in macrovascular complications in the intensive antidiabetic treatment arm. Only in the ADVANCE study a trend was observed towards the reduction in cardiovascular mortality by $12 \%$ in the intensive treatment group. Moreover, the results of the ACCORD study revealed increased mortality in patients who underwent intensive treatment. The reason for this observation still remains unclear; however, the results of above-mentioned trials gave an impulse to change guidelines for the management of type 2 diabetes. It was concluded that the treatment should be far more individualized. For every patient, the risk for hypoglycaemia, the level of diabetes education and the cost-benefits relationship of specific therapy should be considered [9-13].

Type 2 diabetes is one of major cardiovascular risk factors. Epidemiology data indicate that cardiovascular complications are responsible for about $65 \%$ of deaths in type 2 diabetes patients. Additionally, over $60 \%$ of patients diagnosed with stable or unstable coronary artery disease (CAD) have glucose metabolism disturbances, such as impaired fasting glucose (IFG), IGT or overt type 2 diabetes.

Diabetes has been diagnosed in $20-45 \%$ of clinical trial participants, including patients hospitalized for acute coronary syndrome (ACS), who had no history of diabetes. In this group of patients, diabetes is more prevalent in women than in men (41.6 vs. $30.7 \%$ ). Many years ago, Haffner et al. published results from clinical trials that suggested that diabetes should be regarded as a coronary heart disease (CHD) equivalent [10]. The increase in cardiovascular risk in diabetic patients is caused not only by diabetes per se, but also by the presence of other cardiovascular risk factors [14-16].
Therefore, establishing the principles for long-term, safe, well-tolerated and effective therapy in patients with diabetes (mainly cardiovascular) complications of various severity, is a significant issue related to new classes of drugs, such as incretin-based agents. New antidiabetic drugs should not only influence glycaemic control, but also contribute to decrease in cardiovascular morbidity and mortality in patients with type 2 diabetes. Taking the above into account, in December 2008 Science Board of the Food and Drug Administration (FDA) issued a guidance requiring the assessment of cardiovascular risk for all new molecules used for the treatment of type 2 diabetes. This guidance specifies methodology of clinical trials and registration of cardiovascular events defined as mortality due to cardiovascular diseases, non-fatal myocardial infarction and/or ischaemic stroke as well as hospitalization due to acute coronary syndrome and the need for coronary revascularization. It is recommended that these trials should be performed in diabetic patients with increased cardiovascular risk, in patients aged $>65$ years, and in patient with kidney dysfunction, assuming the follow-up period of $\geq 2$ years [17].

These trials are designed to show that the use of antidiabetic drugs as one of the standard option for the treatment of diabetes is not associated with the increase in cardiovascular risk (cardiovascular safety) compared with placebo. However, it should be highlighted that they are not designed to show cardiovascular benefits resulting from $\mathrm{HbA}_{1 \mathrm{c}}$ reduction. Cardiovascular safety and cardiovascular benefits should be assessed regardless of the $\mathrm{HbA}_{1 \mathrm{c}}$ reduction.

DPP-4 inhibitors, also known as gliptins, are a group of oral glucose-lowering drugs whose mechanism of action is based on competitive inhibition of dipeptidyl peptidase-4 activity, and subsequent increase in incretin hormone concentration, mainly GLP-1 and GIP. These hormones stimulate insulin secretion by $\beta$-cells and decrease glucagon concentration in glucose-dependent fashion. They improve glycaemic control, both in fasting state and after meals. Available data from clinical studies indicate possible regeneration of pancreatic $\beta$-cells. There are four DPP-4 inhibitors available in Poland: sitagliptin (Januvia, Merck Sharp \& Dohme), vildagliptin (Galvus, Novartis), saxagliptin (Onglyza, Bristol Myers Squibb/AstraZeneca) and linagliptin (Trajenta, Boehringer Ingelheim).

Given that GLP-1 receptors' expression was found not only in pancreatic islets, but also in the heart, vascular endothelium, gastrointestinal tract, central (hypothalamus) and peripheral nervous systems, lungs and kidneys, DPP-4 inhibitors as a class of antidiabetic drugs offer unique benefits for type 2 diabetes patients that extent beyond the impact on glycaemic control $[18,19]$. 


\section{Effect on body weight}

Obesity is one of the main risk factors for development and progression of type 2 diabetes, but also cardiovascular diseases per se. Many of the drugs currently used for the treatment of type 2 diabetes, induce weight gain (sulphonylureas, thiazolidinediones, insulin). Incretin-based agents are weight-neutral or promote weight reduction [5-7, 19-22].]

\section{Antiatherogenic effect}

DPP-4 inhibitors have beneficial effect on independent cardiovascular risk factors. Besides glucose level fluctuations, in patients with diabetes these factors include postprandial lipid levels, hypertension, obesity, insulin resistance and compensatory hyperinsulinaemia. All these factors increase oxidative stress and epithelial dysfunction, exert prothrombotic and proinflammatory effect, activate parasympathetic system and, as a result, stimulate atherogenesis. DPP-4 inhibitors have favourable effect on lipid profile by reducing the serum concentration of total cholesterol, LDL cholesterol, triglycerides and postprandial lipoproteins reach in triglycerides and increasing the serum concentration of HDL cholesterol. These drug promote the reduction of hs-CRP concentration, decrease plasminogen activator inhibitor-1 (PAI-1) and B-type natriuretic peptide (BNP) activities and increase adiponectin concentration. This effect is independent from body weight reduction [21, 23-25].

\section{Hypotensive effect}

Native GLP-1 exerts diuretic action and increases sodium excretion in response to volume overload and the increase in serum sodium concentration, and therefore it can, to some degree, reduce blood pressure. DPP-4 therapy also results in slightly decreased blood pressure. In published clinical trials, systolic blood pressure reduction ranged from 2 to $5 \mathrm{~mm} \mathrm{Hg}(p<0.05)$, while no influence on diastolic blood pressure was shown [21, 23-25].

\section{Cardioprotective effect}

Cardioprotective action through incretin effect has been shown not only for native GLP-1, but also for GLP-1 (9-36) peptide, a product of GLP-1 (7-36) degradation by DPP-4. It has been found that administration of GLP-1 (9-36) during myocardial reperfusion improved myocardial function in GLP-1 receptor knock-out animal model, providing a significant protection against ischaemic/reperfusion damage, and induced vessel dilation by mechanism associated with nitric oxide/cGMP pathway that does not require active GLP-1 receptor.

In a study assessing the influence of DPP-4 inhibitors on a global function of left ventricle in patients with myocardial ischaemia, it has been found that administration of the drug resulted in a significant improvement in global and regional left ventricular function. Furthermore, myocardial regeneration due to stem cell mobilization was observed in patients after myocardial infarction who were administered DPP-4 inhibitor and granulocyte-colony stimulating factor (G-CSF). Moreover, it has been shown that DPP-4 inhibition promotes blood vessel dilation and peripheral resistance decrease due to the degradation of some vasoactive peptides, such as neuropeptide $Y$ or neuropeptide $P$ [21, 23-26].

\section{Effect on cardiovascular risk}

In 2012, a systemic review was published that included a meta-analysis of 18 randomized clinical trials comprising 8,500 type 2 diabetes patients (among them almost 5,000 patients treated with DPP-4 inhibitor), with a meanfollow-up period of 46 weeks. It was shown that these drugs have beneficial impact on cardiovascular risk in this special population. Relative risk for adverse cardiovascular events (cardiovascular mortality, non-fatal myocardial infarction, acute coronary syndrome, stroke, arrhythmia and heart failure) was significantly lower in patients receiving DPP-4 inhibitor (RR 0.48; 95\% Cl 0.1-0.75, p=0.001) compared with the groups receiving other therapies. Relative risk for non-fatal myocardial infarction or acute coronary syndrome was 0.4 and was significantly decreased compared with other therapies $(95 \% \mathrm{Cl} 0.18-0.88$; $\mathrm{p}=0.02$ ). The authors are however cautious in drawing final conclusions. They suggest that the observed difference was not necessarily related to the risk reduction by DPP-4 inhibitors, but may have resulted from the impact of other comparators on this risk increase (sulphonylureas, thiazolidinediones or metformin) [27].

Therefore, the final answer for the question: 'Whether and to what extent does the therapy with DPP-4 inhibitors influence cardiovascular outcomes?' will depend on the results of the trials designed according to FDA recommendation, aiming to objectively assess the impact of long-term treatment with this class of drugs on pre-defined cardiovascular endpoints.

In 2013, the results of the first of these trials, SAVOR-TIMI 53 (The Saxagliptin Assessment of Vascular Outcomes Recorded in Patients with Diabetes Mellitus-Thrombolysis in Myocardial Infarction), were published. This multicentre, randomized, double-blind trial including 16,492 patients evaluated the efficacy and safety of saxagliptin alone or in combination with other glucose-lowering drugs, including insulin, in type 2 diabetes patients aged $>40$ years, with $\mathrm{HbA}_{1 \mathrm{c}}>6.5 \%$, who had concomitant cardiovascular disease or at least 2 cardiovascular risk factors. The meanfollow-up period 
was 2.1 years. This study showed that saxagliptin added to standard glucose-lowering medication in patients at increased cardiovascular risk did not contribute to reduction or increase of the risk of the composite primary endpoint defined as cardiovascular death, myocardial infarction or ischaemic stroke. Thus, the study demonstrated non-inferiority of saxagliptin versus placebo. However higher rate of hospitalization for heart failure was observed in the saxagliptin group (3.5\% vs. $2.8 \%$; RR 1.27; 95\% Cl, 1.07-1.51; $p=0.007)$. It should be however noted that the patients with the highest risk for hospitalization were previously diagnosed with heart failure, increased concentration of natriuretic peptide or chronic kidney disease.

The rate of hypoglycaemia-related hospitalization was similar in both groups; however, in the saxagliptin group significantly more patients reported at least one episode of hypoglycaemia [1,264 patients (15.3\%) vs. $1,104$ patients $(13.4 \%) ; p<0.001]$. Severe hypoglycaemic episodes were noted in 177 patients (2.1\%) treated with saxagliptin compared with 140 patients $(1.7 \%)$ in the placebo group ( $p=0.047$ ) [28].

Alogliptin was another DPP-4 inhibitor for which the data were published from the cardiovascular safety trial meeting FDA requirements. Multicentre, randomized, placebo-controlled, double-blind EXAMINE (Alogliptin after Acute Coronary Syndrome in Patients with Type 2 Diabetes) trial included 5,380 patients with type 2 diabetes and recent acute coronary syndrome (myocardial infarction or angina pectoris) that occurred within 15-90 days prior to enrolment. The mean follow-up period was 1.5 years. The primary endpoint, defined as cardiovascular death, non-fatal myocardial infarction or non-fatal stroke, occurred with similar frequency in both study groups. No significant differences were shown between the groups in terms of the secondary endpoint, including additionally theneed for revascularization due to unstable angina. The rate of hypoglycaemic episodes was comparable between the group receiving active treatment and in the placebo group. In 2015 the results of post-hoc analysis were published that showed no significant differences in composite endpoint including cardiovascular death and hospitalization due to heart failure. A limitation of this study seems to be relatively short follow-up period [29].

The results of another study determining cardiovascular safety in type 2 diabetics treated with sitagliptin - TECOS (The Trial to Evaluate Cardiovascular Outcomes after Treatment with Sitagliptin) — were also published in 2015. This was, similarly as SAVOR TIMI and EXAMINE, a randomized, double-blind and prospective trial. A total of 14,671 type 2 diabetes patients with baseline cardiovascular complications were followed-up. Composite primary endpoint was defined as cardiovascular death, non-fatal myocardial infarction, non-fatal stroke or hospitalization due to unstable angina. The meanfollow-up of 3 years was longer than in previous trials. No significant differences were found for primary endpoint between sitagliptin treatment and comparator, which demonstrated noninferiority of sitagliptin. Similarly, hospitalization rate due to heart failure was similar in both groups (RR $1.00 ; 95 \% \mathrm{Cl}, 0.83-1.20 ; p=0.98)$. Adding sitagliptin to previous therapy did not increase the risk for hypoglycaemia [30].

Another study, evaluating the most recently registered DPP-4 inhibitor, linagliptin, started in 2010. The CAROLINA (Cardiovascular Outcome Study of Linagliptin Versus Glimepiride in Patients With Type 2 Diabetes) trial is a multicentre, randomized, double-blind and placebo-controlled observation aiming to assess the efficacy and safety of linagliptin therapy in type 2 diabetes patients aged 40-85 years, with documented cardiovascular disease or at least 2 cardiovascular risk factors. The primary and secondary endpoints are similar to those in TECOS, SAVOR-TIMI 53 and EXAMINE trials (cardiovascular death, non-fatal myocardial infarction or stroke, hospitalization due to unstable angina pectoris). The end of the study and publication of its results are planned for 2018. It should be, however, noted that among described above trials only in this one a reference group receives active comparator, glimepiride, rather than placebo. Available randomized controlled trials (RCT) for linagliptin including over 9000 patients with type 2 diabetes show clearly that there is no risk increase not only versus placebo but also, which is particularly important, versus active comparators [31].

\section{Conclusions}

Summing up, it should be highlighted that although DPP-4 inhibitors offer unique benefits for patients with type 2 diabetes extending beyond the impact on glycaemic control, our knowledge of full therapeutic potential of incretin axis, including possible protective role on cardiovascular system, needs further research. Based on the analysis of available data from clinical trials, it may be stated that these drugs reduce blood pressure, increase left ventricular ejection fraction, have favourable effect on lipid profile and improve endothelial function; however, we have to wait for the results of further trials with DPP-4 to receive conclusive answer whether long-term treatment with this class of antidiabetic drugs translates into real reduction of cardiovascular risk. 


\section{REFERENCES}

1. UK Prospective Diabetes Study Group: Intensive blood-glucose control with sulphonylureas or insulin compared with conventional treatment and risk of complications in patients with type 2 diabetes (UKPDS 33). Lancet 1998; 352: 837-853.

2. Koro CE, Bowlin SJ, Bourgeois N, Fedder DO. Glycemic control from 1988 to 2000 among U.S. adults diagnosed with type 2 diabetes: a preliminary report. Diabetes Care 2004; 27: 17-20.

3. Saydah SH, Fradkin J, Cowie CC. Poor control of risk factors for vascular disease among adults with previously diagnosed diabetes. JAMA 2004; 291: 335-342.

4. DeFronzo RA. Pharmacologic therapy for type 2 diabetes mellitus. Ann Intern Med 1999; 131: 281-303.

5. Holst JJ, Gromada J. Role of incretin hormones in the regulation of insulin secretion in diabetic and nondiabetic humans. Am J Physiol Endocrinol Metab 2004; 287: E199-E206.

6. Nauck M, Stockmann F, Ebert R, Creutzfeldt W. Reduced incretin effect in type 2 (non-insulin-dependent) diabetes. Diabetologia 1986; 29: 46-54.

7. Shuster LT, Go VLW, Rizza RA, O’Brien PC, Service FJ. Incretin effect due to increased secretion and decreased clearance of insulin in normal humans. Diabetes 1988; 37: 200-203.

8. Drucker DJ. Enhancing incretin action for the treatment of type 2 diabetes. Diabetes Care 2003; 26: 2929-2940.

9. UK Prospective Diabetes Study (UKPDS) Group. Intensive bloodglucose control with sulphonylureas or insulin compared with conventional treatment and risk of complications in patients with type 2 diabetes(UKPDS 33). Lancet 1998; 352: 837-853.

10. The ADVANCE Collaborative Group. Intensive Blood Glucose Control and Vascular Outcomes in Patients with Type 2 Diabetes. N Engl J Med 2008; 358: 2560-2572.

11. Nilsson PM. ACCORD and Risk-Factor Control in Type 2 Diabetes. N Engl J Med 2010; 362: 1628-1630.

12. Duckworth W, Abraira C, Moritz T, Reda D, Emanuele N. Glucose Control and Vascular Complications in Veterans with Type 2 Diabetes. N Engl J Med 2009; 360: 129-139.

13. The Action to Control Cardiovascular Risk in Diabetes Study Group Effects of Intensive Glucose Lowering in Type 2 Diabetes. N Engl J Med 2008; 358: 2545-2559.

14. Franklin K, Goldberg RJ, Spencer F et al. Implications of diabetes in patients with acute coronary syndromes. The Global Registry of Acute Coronary Events. Arch Intern Med 2004; 164: 1457-1463.

15. Hasdai D, Behar S, Wallentin L et al. A prospective survey of characteristics, treatments and outcomes of patients with acute coronary syndromes in Europe and Mediterranean basin; the Euro Heart Survey of Acute Coronary Syndromes (Euro Heart Survey ACS). Eur Heart J 2002; 23: 1190-1201.

16. Haffner SM, Lehto S, Rönnemaa T, Pyörälä K, Laakso M. Mortality from coronary heart disease in subjects with type 2 diabetes and in nondiabetic subjects with and without prior myocardial infarction.N Engl J Med. 1998; 339: 229-234.

17. US FDA. Guidance for Industry Diabetes Mellitus — Evaluating Cardiovascular Risk in New Antidiabetic Therapies to Treat Type 2 Diabetes, (December), 2008. http://www.fda.gov/downloads/drugs/guidancecomplianceregulatoryinformation/guidances/ucm071627.pdf.

18. Hinnen D, Nielsen LL, Waninger A, Kushner A. Incretin Mimetics and DPP-IV Inhibitors: New Paradigms for the Treatment of Type 2 Diabetes. J Am Board Fam Med 2006; 19: 612-620.

19. Ahre' $n$ B, Landin-Olsson $M$, Jansson $P$, Svensson $M$, Holmes $D$, Schweizer A. Inhibition of dipeptidyl peptidase-4 reduces glycemia, sustains insulin levels, and reduces glucagon levels in type 2 diabetes. J Clin Endocrinol Metab 2004; 89: 2078-2084.

20. Pratley RE, Gilbert M. Targeting Incretins in Type 2 Diabetes: Role of GLP-1 Receptor Agonists and DPP-4 Inhibitors. Rev Diabet Stud 2008; 5: 73-94.

21. Ussher JR, Drucker DJ. Cardiovascular Biology of the Incretin System. Endocrine Reviews 2012; 33: 187-215.

22. Amori RE, Lau J, Pittas AG. Efficacy and safety of incretin therapy in type 2 diabetes: systematic review and meta-analysis. Journal of the American Medical Association 2007; 298: 194-206.

23. Simsek S, de Galan BE. Cardiovascular protective properties of incretin-based therapies in type 2 diabetes. Curr Opin Lipidol 2012; 23: 540-547.

24. Mannucci E, Dicembrini I. Incretin-based therapies and cardiovascular risk. Curr Med Res Opin 2012; 28: 715-721.

25. Matsubara J, Sugiyama S, Akiyama E et al. Dipeptidyl peptidase-4 inhibitor, sitagliptin, improves endothelial dysfunction in association with its anti-inflammatory effects in patients with coronary artery disease and uncontrolled diabetes. Circulation Journal 2013; 12: 1168.

26. Shah Z, Pineda C, Kampfrath T et al. Acute DPP-4 inhibition modulates vascular tone through GLP-1 independent pathways. Vascular Pharmacology 2011; 55: 2-9.

27. Patil HR, Al Badarin FJ, Shami HA et al. Meta-Analysis of Effect of Dipeptidyl Peptidase-4 Inhibitors on Cardiovascular Risk in Type 2 Diabetes Mellitus. Am J Cardiol 2012; 15: 826-833.

28. Scirica, BM, Bhatt DL, Braunwald E et al Saxagliptin and cardiovascular outcomes in patients with type 2 diabetes mellitus The New England Journal of Medicine 2013; 369: 1317-1326.

29. White WB, Cannon CP, Heller SR et al. Alogliptin after Acute Coronary Syndrome in Patients with Type 2 Diabetes. N Engl J Med 2013; 369: 1327-1335.

30. Green JB, Bethel MA, Armstrong PW et al. Effect of Sitagliptin on Cardiovascular Outcomes in Type 2 Diabetes. N Engl J Med. 2015; 373: 232-242.

31. Rosenstock J, Marx N, Neubache D et al. Cardiovascular safety of linagliptin in type 2 diabetes: a comprehensive patient-level pooled analysis of prospectively adjudicated cardiovascular events. Cardiovasc Diabetol 2015; 14: 57. doi: 10.1186/s12933-015-0215-2. 